\title{
Effective Throughput for Coded OFDM/SDMA Systems with Pilot-Assisted Channel Estimation
}

\author{
Dongxu Shen, Kai-Kit Wong, Zhengang Pan, and Victor O. K. Li \\ Department of Electrical \& Electronic Engineering \\ The University of Hong Kong \\ Pokfulam Road, Hong Kong, China \\ email: \{dxshen, kitwong, zgpan, vli\}@eee.hku.hk
}

\begin{abstract}
Ahstract-This paper investigates the performance of coded orthogonat frequency division multiplexing (OFDM) systems with receiving adaptive atris, through which multiple users with single-element transmitting antena are supported simultaneously by spatial division multiple access (SI)MA). We characterize the performance of an OFDM/SDMA systems by effective honghpu, which is essentially the average number of datu bits in an Olil symbol after considering the erroneous packet transmissions and modulation scheme by excluding the overhead from coding and pilots for channel estimation. Optimization of system operating parameters can be achieved through the maximization of effective throughput. The focus of this paper is to study the impact of pilot density and the number of users on the performance of coded OFDM/SDMA systems. Through extensive computer simulation, we show that using more pilots always improves bit error rate (BER) performance, hut may reduce effective tlroughput. The optimal number of pilots together with the modulation scheme can be determined by maximizing the effective throughput for given operating signal-to-noise ratio (SNR). It is also shown that the system performance degrades gradwally with the increase of users. For a system with a six-element adaptive arais, the effective throughput with 5 users is lower than that with 4 users for a certain range of $S$ NR. This indicates that the maximal number of user: supportable by the system should consider the effective throughput.
\end{abstract}

\section{INTRODUCTION}

Orthosonal frequency division multiplexing (OFDM) [1] is a promising technology for high speed data communications, and has been adopted in wireless local area networks (LAN) standards such as IEEE 802.11 a [2] and HIPERLAN II [3]. While OFDM is capable of combating inter-symbol interference (ISI), its performance can still be severely degraded by channel fading. An cffective method to mitigate the influence of fading is to employ adaptive array [4], [6], through which multiple simultaneous users can further be supported by spatial division multiple access (SDMA).

In this paper, we study the uplink performance of an coded OFDM/SDMA wireless communication system. The base station (BS) is equipped with an adaptive antenna array for signal reception, and each mobile user transmits through a singleelement antenna.

The performance of coded OFDM/SDMA systems can be characterized by effective throughput proposed in [5], which essentially represents the average number of data bits carried in an OFDM symbol after considering the modulation scheme and packet error while excluding the overhead from channel estimation and coding. Therefore, the optimization of system performance is equivalent to the maximization of effective throughput. Through the characterization by effective throughput, the influence of various factors on system performance can be quantified.

In this paper, the focus is on studying how the system performance is influenced by the pilot-aided channel estimation [8], modulation, coding schemes, and the number of simultancously transmitting users. Through simulation, we show that it is not always beneficial to have more pilots for channel estimation, because the use of pilots reduces the throughput for data. The optimal number of pilots should be determined together with the modulation scheme in order to maximize the effective throughput given the operating signal-to-noise ratio (SNR). Similarly. supporting more users may result in the reduction of effective throughput. In particular, simulation results demonstrate for a system with a six-element adaptive array, the effective throughput with 5 users can be lower than that with 4 users for a certain range of SNR. This indicates that the maxinal number of users supportable by the system is not only limited by the freedom of the system (number of receiving antenna elements), but is also conditioned upon effective throughput and operating SNR.

This paper is organized as follows. In Section II, we describe the system. The definition of effective throughput is presented in Section III. In Section IV, the performance of an OFDM/SDMA system is investigated. We conclude this paper in Section $V$.

\section{SYSTFM DESCRIPTION}

The baseband processing of a mobile user $u, u=1 \ldots, U$ is depicted in Fig. 1. We assume the number of simultaneous users $U$ is no greater than the number of antenna elements $A$ at the BS. Data bits are first encoded by a convolutional encoder, and interleaved by a random interleaver. The conventional block interleaver is inappropriate because convolutional code performs unsatisfactorily with bursty errors [10] due to the correlated fading on adjacent subcarriers over both time and frequency. The interleaved binary bits are then mapped to a modulation symbol using quadrature amplitude modulation (QAM). After seria!-toparallel conversion, inverse fast Fourier transform (IFFT) is performed on the symbols $S_{u}(i), i=1, \ldots N_{c}$ where $i$ denotes the subcarrier index and $N_{c}$ is the number of subcarriers in an OFDM symbol. The outputs from the IFFT transformer correspond to the time samples of the transmitting signal and are parallel-to-serial converted. After cyclic prefix is added, the resulting signal is finally transmitted from a single-element antenna.

At the BS (sce Fig. 2), signal is received by $A$ antenna elements. We assume the channels between a user and every antenna element are uncorrelated, and signals from different uscrs are independent, which leads to a full diversity system. After sampling and removing cyclic prefix, the samples are serial-to-parallel converted, and demodulated by the fast Fourier 


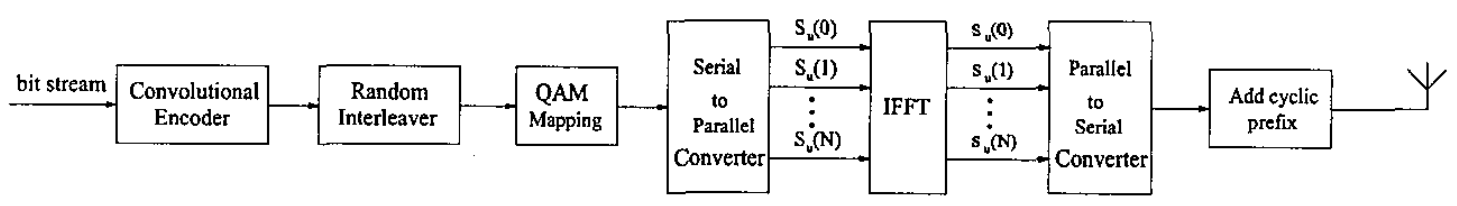

Fig. 1. Baseband processing of a mobile user.

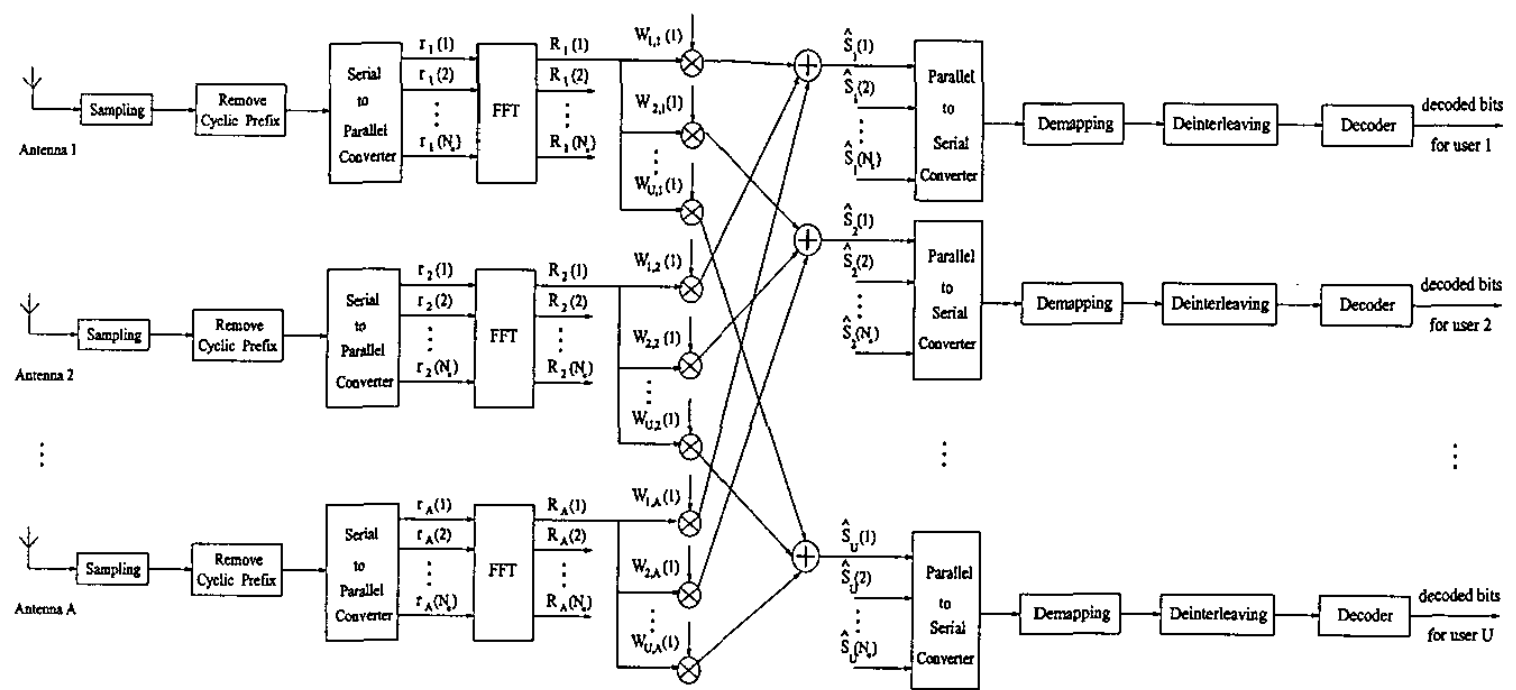

Fig. 2. Baseband processing at the base station. We only show the antenna processing on subcarrier 1 , while the processing on other subcarriers is identical and is omitted.

transform (FFT) processor to produce $R_{a}(i), a=1, \ldots A$, $i=1, \ldots, N_{c}$ at antenna $a$. For subcarrier $i$, the signals from different antenna elements $R_{a}(i), a=1, \ldots, A$ are weighted and combined to form an estimate $\widehat{S}_{u}(i), u=1, \ldots, U$ for the transmitted signal $S_{u}(i), u=1, \ldots, U$. The estimated signal is finally mapped back to binary bits, which are then deinterleaved and decoded.

To illustrate how the antenna processing works, let $\mathbf{S}(i)=$ $\left[S_{1}(i), \ldots, S_{U}(i)\right]^{T}$ be the vector of transmitted signal on subcarrier $i$, where $T$ denotes the transpose operation. Also, let $\mathrm{R}(i)=\left[R_{1}(i), \ldots, R_{A}(i)\right]^{T}$ be the received signal vector, and $\mathbf{n}(i)=\left[n_{1}(i), \ldots, n_{A}(i)\right]^{T}$ be the noise vector, where $n_{a}(i)$ is the noise on subcarrier $i$ at antenna $a$. Since users are assumed to be perfectly synchronized in both time and frequency, the received signal for subcarrier $i$ is expressed as

$$
\mathbf{R}(i)=\mathbf{H}(i) \mathbf{S}(i)+\mathbf{n}(i), \quad i=1, \ldots, N_{c}
$$

where $\mathbf{H}(i)$ is the channel transfer matrix on subcarrier $i$

$$
\mathbf{H}(i)=\left[\begin{array}{ccc}
H_{1.1}(i) & \cdots & H_{U .1}(i) \\
\vdots & \ddots & \vdots \\
H_{1, A}(i) & \cdots & H_{U, A}(i)
\end{array}\right]
$$

with $H_{u . a}(i)$ being the fading coefficient between user $u$ and antenna element $a$ at the BS. Denoting the antenna weight matrix as

$$
\mathbf{W}(i)=\left[\begin{array}{ccc}
W_{1,1}(i) & \cdots & W_{U .1}(i) \\
\vdots & \ddots & \vdots \\
W_{1, A}(i) & \cdots & W_{U, A}(i)
\end{array}\right]
$$

where $W_{u, a}(i)$ is the antenna weight for the $i$ th subcarrier of user $u$ at antenna $a$, the transmitted signal vector $\mathbf{S}(i)$ can be estimated by

$$
\widehat{\mathbf{S}}(i)=\mathbf{W}^{H}(i) \mathbf{R}(i)
$$

where $H$ represents the Hermitian transpose.

With channel state information, the optimal weight matrix W(i) can be computed by [9]

$$
\mathbf{W}(i)=\left[\mathbf{H}(i) \mathbf{H}^{H}(i)+\sigma^{2} \mathbf{I}\right]^{-1} \mathbf{H}(i), i=1, \ldots, N_{c},
$$

where $I$ is an $A \times A$ identity matrix. In this paper, the channel state information is obtained through the use of pilots [8].

\section{EFFECTIVE Throughput}

We define the effective throughput in this section. This concept is devised to characterize the performance of an OFDM/SDMA system by considering the packet error rate (PER), overheads from pilots, modulation scheme and coding rate.

Definition 1-User Effective Throughput: Let $P_{e}$ be the PER, $N_{d}$ the number of data subcarriers in an OFDM symbol, $M_{I}$ the 
modulation index on data subcarriers, and $r_{c}$ the coding rate. The effective throughput for a user is defined as

$$
T_{i l} \triangleq\left(1-P_{e}\right) N_{d} M_{I} r_{C}
$$

The nomalized effective throughput is defined as the ratio beiween the user effective throughput and the number of subcarriers in an OFDM symbol. That is

$$
\begin{aligned}
\bar{T}_{u} & \triangleq \frac{T_{u}}{N_{t}} \\
& =\frac{\left(1-P_{c}\right) N_{d} M_{t} r_{t}}{N_{t}} .
\end{aligned}
$$

Definition 2-System Effective Throughput: Let $T_{u}(i)$ be the effective throughput for user $i$. When there are $U$ users transmitting simultaneously, the system effective throughput is defined as

$$
T_{a} \triangleq \sum_{i=1}^{U} \Gamma_{u}(i) .
$$

The normalized system effective throughput is similarly defined as

$$
\begin{aligned}
\bar{I}_{i} & \triangleq \sum_{i=1}^{v} \vec{T}_{u}(i) \\
& =\frac{T_{i x}}{N_{1}} .
\end{aligned}
$$

\section{Discussions and Simulation Results}

In this section, we discuss the impact of various factors on the performance of coded OFDM/SDMA through simulation, with the emphasis on the pilot density and number of users. The performance criterion we use is the normalized effective throughput for a user, but sometimes BER is used as a supplement. Throughout this section, we shall use the term effective throughput interchangeably with normalized effective throughput for convenience.

\section{A. Simulation Setup}

We assume all users apply the same operating parameters, and the channels between every user and the BS are statistically independent and identical. Therefore, all users would have statistically the same performance.

The multipath fading channel is assumed to be wide sense stationary with uncorrelated scattering (WSSUS). In the time domain. channel impulse response is modeled as a tapped delay line at tap spacing $T_{s}$, with each path following an exponential power delay profile. The amplitude on each path is Rayleigh distributed, and the phase is uniformly distributed between $[0,2 \pi]$. The method in [11] is used for the simulation of channel fading. The simulation parameters are summarized in Table $\mathrm{I}$.

in our simulation. pilots are inserted for channel estimation. To confine the overheads from pilots. only onc of every $M$, OFDM symbols is inserted with pilots on subcarriers that arc $M_{f}$ subcarriers apart, and the arrangement is illustrated in Fig. 3. When a subcarrier is used as a pilot subcarrier for a user. other users place null symbols on the subcarrier to avoid interference, i.c., no signal (neither pilot nor data) is carried by the subcarrier. After estimating the channel on pilot subcarriers in a pilot-bearing OFDM symbol, the channels on all
TABL.EE I

PARAMETERSFOR AN OFDM/SDMA SYSTEM.

\begin{tabular}{|c|c|}
\hline Number of antennas & $A=6$ \\
\hline Number of users & $U=3.4$ or 5 \\
\hline Carricr frequency & $f_{c}=5.4 \mathrm{GHz}$ \\
\hline Bandwidth & $20 \mathrm{MHz}$ \\
\hline Number of subcarricrs & $N_{\mathrm{c}}=128$ \\
\hline Carrier spacing & $\Delta f=20 / 128=162.5 \mathrm{kHz}$ \\
\hline Sampling interval & $T_{s}=1 / N \Delta f=0.05 \mu \mathrm{s}$ \\
\hline FFT period & $T_{F}=1 / \Delta f=6.4 \mu \mathrm{s}$ \\
\hline Guard interval & $T_{G}=0.8 \mu \mathrm{s}$ \\
\hline Symbol interval & $T_{S}=T_{F}+T_{G}=7.2 \mu \mathrm{s}$ \\
\hline Packet length & 500 bytes \\
\hline Modulation schemes & $\mathrm{QPSK}, 16-\mathrm{QAM}, 64-\mathrm{QAM}$ \\
\hline Coding scheme & Convolutional code \\
\hline Coding rate & $r_{c}=1,1 / 2,1 / 3,1 / 4$ \\
\hline Gcnerator functions & $(753,561),(557,663.771)$ \\
(in octals) & $(765,671,513.473)$ \\
\hline Interleaver & Random interlcaver \\
\hline Channel delay spread & $0.8 \mu \mathrm{S}$ \\
\hline Doppler frequency & $F_{d}=1389 \mathrm{~Hz}$ \\
\hline Pilot density & $M_{f}=4$ or $8, M T_{t}=5$ \\
\hline Pilot SNR & $20 \mathrm{~dB}$ \\
\hline
\end{tabular}

other subcarriers of that OFDM symbol are estimated by a maximal likelihood (ML) estimator [12]. Then linear interpolation is performed based on the estimation from two consecutive pilot-bearing OFDM symbols for estimating the channels of the $M_{t}-1$ OFDM symbols in between [13]. To ensure the quality of channel estimation, we require the SNR on the pilot subcarriers to be always maintained at $20 \mathrm{~dB}$ regardless of the $S N R$ on data subcarriers.

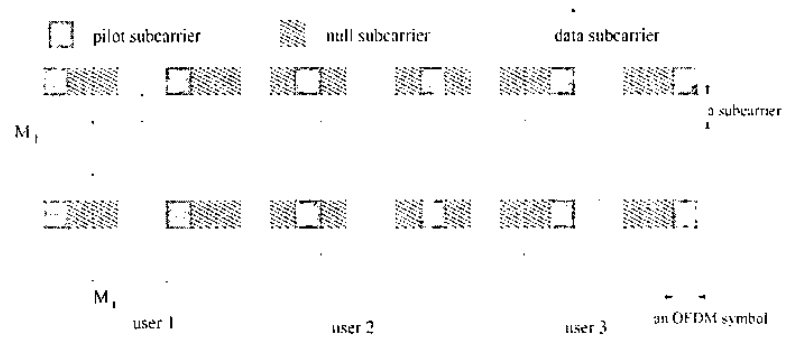

Fig. 3.. An example for pilot arrangement with three users.

\section{B. Pilot Density}

We first compare the performance under different number of pilots. In the simulation, $M_{t}$ is fixed at $5, M_{f}$ is 4 or 8 and $U=3$.

The BER for the uncoded QPSK, 16-QAM, and 64:QAM with $M_{f}=4$ or $8, M_{t}=5, U=3$ is plotted in Fig. 4. From Fig. 4 , we notice that by doubling the pilot density in the frequency domain (from $M_{f}=8$ to $M_{f}=4$ ), there is consistently about $2 \mathrm{~dB}$ gain in SNR for all modulation schemes under investigation. At the same $M_{f}$ and $M_{t}$, there is 6 to $8 \mathrm{~dB}$ difference 


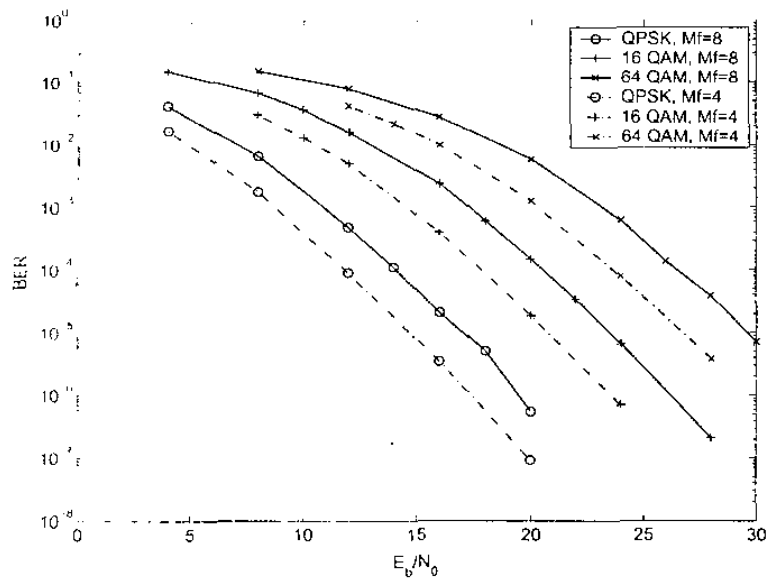

Iiig. 4. I3ER of uncoded QPSK, 16-QAM, and 64-QAM with $M_{f}=4$ or 8 . $M_{t}=5, u=3$

in SNR between QPSK and 16-QAM for the same BER, and 4 to $6 \mathrm{~dB}$ difference between 16-QAM and 64-QAM.

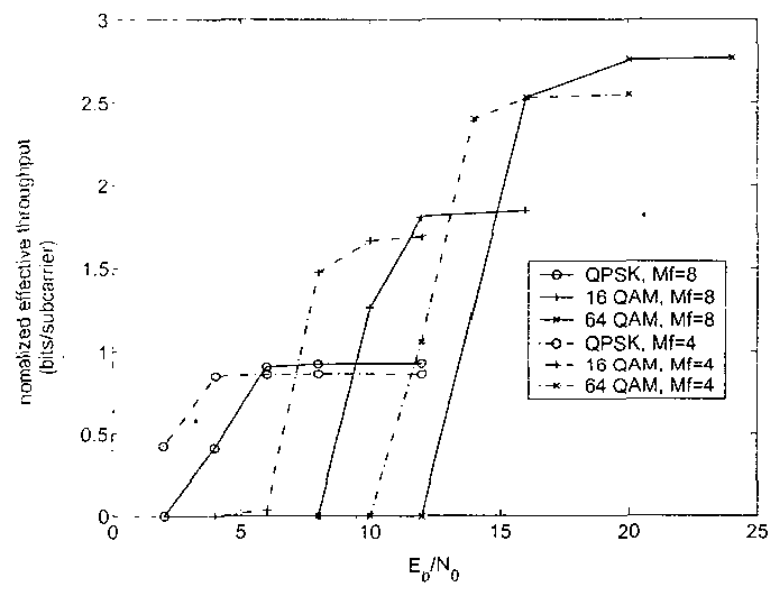

Fig. 5. Eftective throughisu for rate- ${ }_{2}^{1}$ coded QPSK, 16-QAM, and 64 QAM wrth $M M_{f}=4$ or $8 . M_{t}=5, U=3$

The effective throughput for a rate- $\frac{1}{2}$ coded system with $H_{j}=4$ or $8 . M_{t}=5, U=3$ is plotted in Fig. 5. We first observe from Fig. 5 that the curve of effective throughput can be characterized by three segments depending on the SNR. When SNR is lower than a certain threshold, effective throughput is zero. When SNR is higher than a certain cutoff value, effective throughput becomes saturated. Between the two values, effective throughput curve is almost linear. Further, the effective throughput with $M_{f}=4$ is higher than that with $M_{f}=8$ before it becomes saturated, and is lower than that with $M_{f}=8$ in the saturation segment.

The above phenomena can be explained as follows. The normalized effective throughput is expressed in (7) as $\bar{T}_{u}=$ $\left(1-P_{c}\right) N_{d} M_{l} r_{c} / N_{c}$, where $N_{d}$ is the averagc number of data subcarricrs in an OFDM symbol. From our arrangement of pi- lots, $N_{d}$ can be calculated as

$$
N_{d}=N_{c}-N_{c} / M_{f} / M_{t}
$$

Thus when SNR is low, $P_{e} \approx 1$, so that $\bar{T}_{t} \approx 0$; when SNR is high, $P_{e} \rightarrow 0$, so that $\bar{T}_{u}$ is close to the upper linited

$$
N_{d} M_{l} r_{c} / N_{c}
$$

Obviously the limit with $M_{f}=8$ is higher than that with $M_{f}=$ 4. Although more pilots always improves the BER performance. it increascs overheads and thus reduces effective throughput, as shown in Fig. 5.

To maximize effective throughput, the optimal number of pilots can be selected together with the modulation scheme from the effective throughput curves in Fig. 5 when given the operating SNR. For example, when SNR is $10 \mathrm{~dB}$. 16-QAM with $M_{f}=4$ is used; when SNR is $20 \mathrm{~dB}, 64-\mathrm{QAM}$ with $M_{f}=8$ is chosen.

\section{Number of Users}

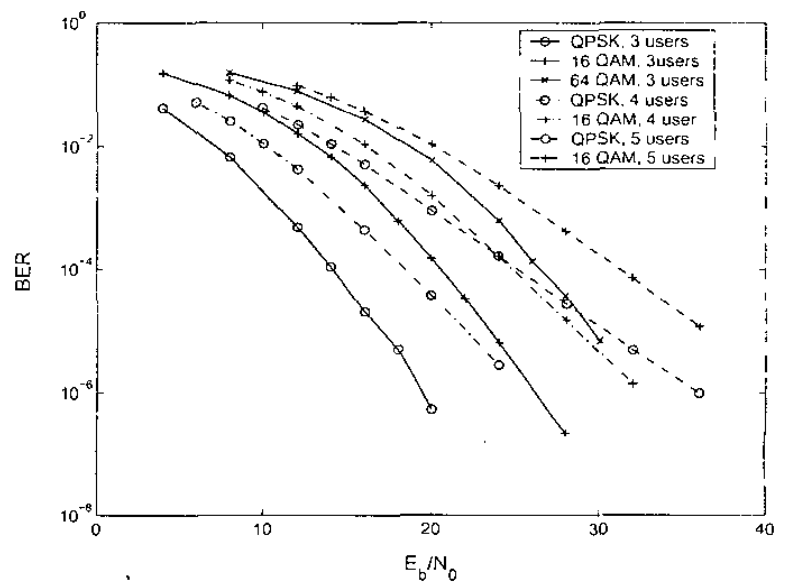

Fig. 6. BER for uncoded QPSK. 16-QAM and 64-QAM wilh 3, 4 and 5 users. $M_{f}=8 \cdot M_{t}=5$.

We investigate the performance impact by the number of users. According to [9], an $A$-clement antenna array has $A-1$ degrees of freedom. To cancel the co-channel interference $(\mathrm{CCI})$ from a uscr, one degree of antenna freedom is needed. As a result, with $U$ users ( $U \leq A$ ), $U$ degrecs of freedom are necded for $\mathrm{CCl}$ cancellation, so that the degree of freedom for diversity is $A-U$. Thus the degree of diversity diminishes with more users.

We plot the BER performance of uncoded systems in Fig. 6. and the corresponding uncoded effective throughput in Fig. 7. It is clear that both the BER and effective throughput performance degrades with more users.

An interesting observation from Fig. 7 is that the effective throughput for uncoded 16-QAM with 4 users is always higher than that of 5 users using either QPSK or 16-QAM. We can also calculate from the definition in (9) that the system effective throughput with 5 users is lower than that with 4 users when 


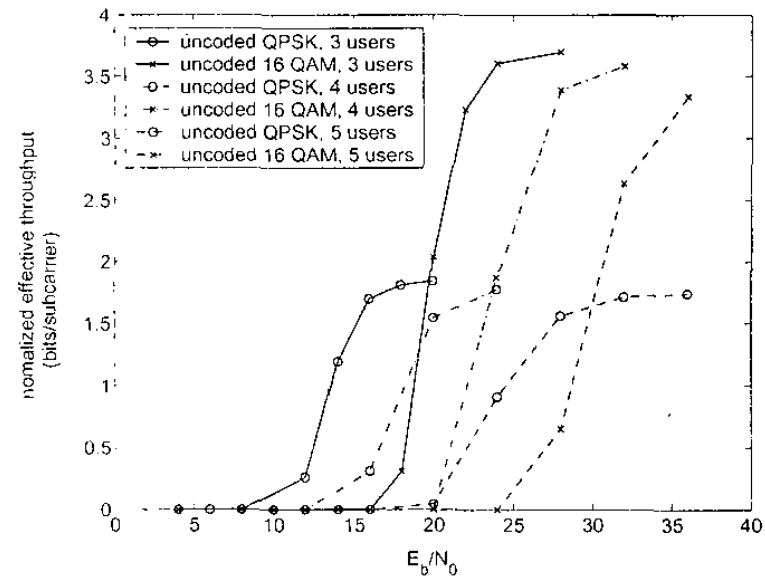

Fig. 7. Eflective throughput for uncoded QPSK, 16-QAM and 64-QAM with 3. 4 ind 5 users. $M_{j}=8, M_{t}=5$.

SNR is lower than $35 \mathrm{~dB}$. For example, when SNR is $32 \mathrm{~dB}$, the effective throughput for a user is about 3.6 bits/subcarrier for 16-QAM when there are 4 users, and is about 2.7 bits/subcarrier for 16-QAM when there are 5 users. The corresponding systen effective throughput is $3.6 \times 4=14.4 \mathrm{bits} / \mathrm{subcarrier}$ and $2.7 \times 5=13.5 \mathrm{bits} / \mathrm{subcarrier}$ respectively. This reveals that the addition of a user can degrade the effective throughput performance for a user and the system at the same time, which is obviously undesirable.

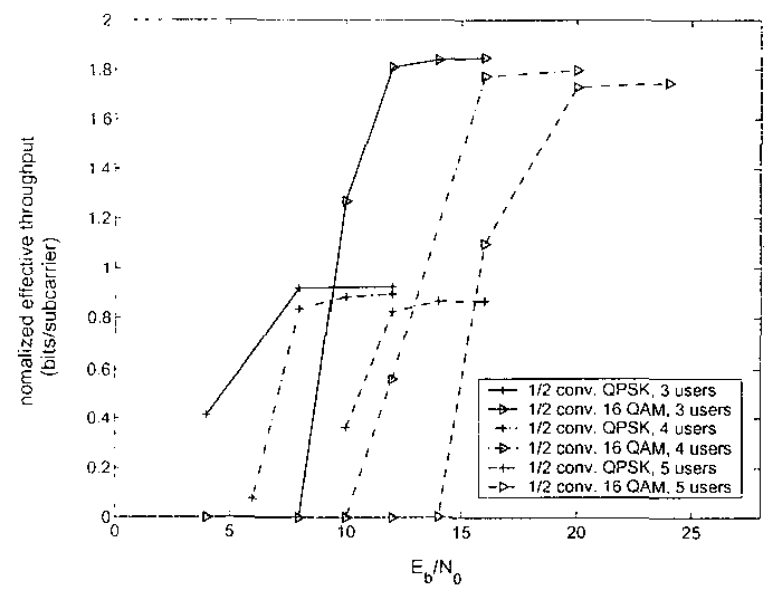

Fig. 8. Fiffective throughput for rate-1/2 coded QPSK and 16-QAM with 3, 4. and 5 users, $M_{f}=8, M_{t}=\overline{3}$.

The coded performance for effective throughput is plotted in Fig. 8. The effective throughput with 5 users employing QPSK is better than that of 4 users employing 16-QAM when SNR is lower than $16 \mathrm{~dB}$, which is much improved as compared with the uncoded case. However, when SNR is above $16 \mathrm{~dB}$, the effective throughput for 5 user with either QPSK or 16-QAM is still lower than that of 4 users with 16-QAM.

\section{CONCLUUSION}

In this paper, we study the performance of coded OFDM/SDMA systems through effective throughput. Specifically, the focus is on investigating the influence of pilot density and number of users. We show that the BER performance improves consistently with more pilots, but the effective throughput can be reduced due to the overhead from pilots. The optimal number of pilots can be determined together with the modulation scheme by maximizing effective throughput at the operating SNR. Simulation results also show that system performance degrades with more uscrs. It is possible for the effective throughput for a user and the system be reduced at the same time by the addition of a user. Thus the maximal number of users that can be supported by an OFDM/SDMA system should be carefully chosen by considering effective throughput.

\section{ACKNOWLEDGMENT}

This research is supported in part by the Research Grants Council of Hong Kong under grant no. HKU 7047/00E.

\section{REFERENCES}

[1] R. van Nee. R. Prasad. OFDM for Wireless Multimedia Communications. Artech House, 2000.

[2] Wireless LAN Medium Access Control (MAC) and Physical Layer (PHY) specification: High-speed Physical Layer in the 5 (iHz Band, IEEE std 802.11 a-1999.

[3] Broadband Radio Access Networks (BRAN): High Performance Radio Local Area Networks (HIPERLAN) Type 2. System Overview, ETSITR $101683 . v 0.1 .2,1999$

[4] K. K. Wong, R. Cheng. K. B. Letaief, R. D. Murch. "Adaptive antennas at the mobile and base stations in an OFDM/TDMA system," IEFE Trans: on Commun., vol. 49, pp. 195-206. Jan. 2001.

[5] D. Shen, Z. Pan, K. K. Weng, V. O. K. Li, "Effective throughput: A unifi ed benchmark for pilot-aided OFDM/SDMA wireless communication systems," Proc. IEEE INFOCOM 201/3, Mar. 2003. San Francisco, Calit..

[6] P. Vandenameele, L. Van Der Perre. M. Engels. B. Gyselinckx. H. De Man "A combined OFDM/SDMA approach." HEEE J. Select. Areas Commm. vol. 18, pp. 2312-2321, Nov. 2000

[7] C. Ganier, M. Loosvelt, V. Le Thuc. Y. Delignon. L. Clavier, "Performance of an OFDM-SDMA based syslem in a time-varying multi-path channel." Proc. ITC 2001. Fall, 2001, vol. 3. $1686-1600$

[8] J. Cavers. "An analysis of pilot symbol assisted modulation for Rayleigh tading channels (mobile radio)." IEEE Trans. Veh. Technol., vol. 40. no. 4. pp. 686-693. 1991.

[9] R. T. Compton, Adaptive Antemas: Concepts and Performance. PrenticeHall, 1988.

[10] S. Lir. D. J. Costello, Jr., Error Conrol Coding: Fundamentals and Applications, Prentice-Hall, 1983

[II] P. Dent, G. E. Bottomley, T. Croft, "Jakes fading model revisited." Electron. Lett. vol. 29, no. 3, pp. 1162-1163, June 1903.

[12] M. Morelli. U. Mengali, "A comparison of pilot-aided channel estimation methods for OFDM systems." IEEE Trans. Signal Processing. vol. 49. no. 12. pp. 3065-3073, Dec. 2001

[13] M. Garcia, J. Paez-Borrallo. S. Zazo. "DFT-based channel estimation in 2D-pilot-symbol-aided OFDM wireless systems." Proc, $V / C \cdot 2001$, Spring, 2001, vol. 2. 810-814. 